\title{
Abordaje transconjuntival en comparación con abordaje subciliar para el tratamiento de fracturas orbitarias.
}

\section{Transconjunctival versus subciliary approach for the treatment of orbital fractures.}

\author{
Juan Pablo Vargas-Buratovic ${ }^{1,2}$, Macarena Uribe-Monasterio ${ }^{1,2}$, Duniel Ortuño-Borroto ${ }^{1,2}$, \\ Francisca Verdugo-Paiva ${ }^{2,3}$, Francisco Javier Pinedo-Henríquez ${ }^{1,4^{*}}$
}

\author{
1. Escuela de Odontología, Facultad de Medicina, \\ Pontificia Universidad Católica de Chile, Santiago, \\ Chile \\ 2. Proyecto Epistemonikos, Santiago, Chile \\ 3. Centro Evidencia UC, Facultad de Medicina, \\ Pontificia Universidad Católica de Chile, Santiago, \\ Chile \\ 4. Hospital Santiago Oriente - Dr. Luis Tisné \\ Brousse, Santiago, Chile.
}

* Correspondencia Autor: Francisco Javier PinedoHenríquez | Dirección: Centro Evidencia UC, Pontificia Universidad Católica de Chile, Diagonal Paraguay 476, Santiago, Chile | E-mail: francisco. pinedo@uc.cl

\section{RESUMEN}

Introducción: Los abordajes transconjuntival preseptal y subciliar han sido ampliamente utilizados para el manejo quirúrgico de las fracturas orbitarias. Sin embargo, aún existe incertidumbre sobre las complicaciones asociadas a cada uno de estos abordajes. Métodos: Realizamos una búsqueda en Epistemonikos, la mayor base de datos de revisiones sistemáticas en salud, la cual es mantenida mediante el cribado de múltiples fuentes de información, incluyendo MEDLINE, EMBASE, Cochrane, entre otras. Extrajimos los datos desde las revisiones identificadas, analizamos los datos de los estudios primarios. Realizamos un metanálisis y preparamos una tabla de resumen de los resultados utilizando el método GRADE. Resultados y conclusiones: Identificamos seis revisiones sistemáticas que en conjunto incluyeron 21 estudios primarios, de los cuales cuatro corresponden a ensayos aleatorizados. Concluimos que el abordaje transconjuntival preseptal podría disminuir tanto la incidencia de ectropión como de un resultado estético insatisfactorio, pero la certeza de la evidencia es baja. Además, este abordaje probablemente disminuye el riesgo de complicaciones intra y postoperatorias, tales como diplopía, parestesia transitoria, equimosis, exposición escleral, laceración del plato tarsal y laceración palpebral inferior. Por otro lado, el abordaje transconjuntival podría aumentar el riesgo de entropión, pero la certeza de la evidencia también es baja.

\begin{abstract}
Introduction: The preseptal transconjunctival and subciliary approach have been widely used for the surgical management of orbital fractures. However, there is still uncertainty about the complications associated with each of these approaches. Methods: We searched in Epistemonikos, the largest database of systematic reviews in health, which is maintained by screening multiple information sources, including MEDLINE, EMBASE, Cochrane, among others. We extracted data from the systematic reviews, reanalyzed data of primary studies, conducted a meta-analysis and generated a summary of findings table using the GRADE approach. Results and conclusions: We identified six systematic reviews that included 21 primary studies, four of which correspond to randomized trials. We conclude that the preseptal transconjunctival approach could decrease the incidence of ectropion and an unsatisfactory aesthetic result, but the certainty of the evidence is low. Furthermore, this approach probably decreases the risk of intra and postoperative complications, such as diplopia, transient paresthesia, ecchymosis, scleral show, tarsal plate laceration and lower palpebral laceration. On the other hand, the transconjunctival approach could increase the risk of entropion, but the certainty of the evidence is also low.

KEY WORDS

Surgical reconstruction; Orbital floor fracture; Transconjunctival approach; Subciliary approach; Epistemonikos; GRADE.
\end{abstract}

Int. J. Inter. Dent Vol. 13(2); 105-109, 2020. 


\section{Mensajes clave}

- El abordaje transconjuntival preseptal en comparación con el abordaje subciliar podría disminuir el riesgo de ectropión y el riesgo de un resultado estético insatisfactorio por parte del paciente (baja certeza de evidencia)

- El abordaje transconjuntival preseptal en comparación con el abordaje subciliar podría aumentar el riesgo de entropión (baja certeza de evidencia)
- El abordaje transconjuntival preseptal, comparado con el abordaje subciliar, probablemente disminuye el riesgo de complicaciones intra y postoperatorias, tales como diplopía, parestesia transitoria, equimosis, exposición escleral, laceración del plato tarsal y laceración palpebral inferior.

\section{PROBLEMA}

Las fracturas orbitarias y orbitocigomáticas constituyen un evento frecuente tras el trauma de la región maxilofacial en pacientes adultos ${ }^{(1)}$. Entre las distintas alternativas para su abordaje existen las de tipo transcutáneo y transconjuntival(2). Dentro del primer grupo, el abordaje más utilizado es el subciliar, mientras que en el segundo grupo el más empleado es el abordaje transconjuntival preseptal(2). La elección entre éstos ha sido un tópico controversial y refleja un balance entre la necesidad de una adecuada exposición del rasgo de fractura y la obtención de una cicatriz aceptable ${ }^{(3)}$.

La complicación más común asociada al uso de estos abordajes corresponde a la malposición palpebral inferior, pudiendo observarse como ectropión o eversión palpebral, entropión o inversión palpebral, exposición escleral, entre otras ${ }^{(2,4,5)}$.

Cada uno de estos abordajes ha sido asociado con ciertas complicaciones específicas y la selección de una técnica u otra podría estar influenciada por la preferencia y comodidad del cirujano tratante ${ }^{(3)}$.

Pese a que se ha intentado demostrar la superioridad de una técnica por sobre otra, aún existe incertidumbre respecto a cuál abordaje es el más efectivo y seguro.

\section{MÉTODOS}

Realizamos una búsqueda en Epistemonikos, la mayor base de datos de revisiones sistemáticas en salud, la cual es mantenida mediante búsquedas en múltiples fuentes de información, incluyendo MEDLINE, EMBASE, Cochrane, entre otras. Extrajimos los datos desde las revisiones identificadas y analizamos los datos de los estudios primarios. Con esta información, generamos un resumen estructurado denominado FRISBEE (Friendly Summaries of Body of Evidence using Epistemonikos), siguiendo un formato preestablecido, que incluye mensajes clave, un resumen del conjunto de evidencia (presentado como matriz de evidencia en Epistemonikos), metanálisis del total de los estudios cuando sea posible, una tabla de resumen de resultados con el método GRADE y una sección de otras consideraciones para la toma de decisión.

\section{Acerca del conjunto de evidencia para esta pregunta}

\begin{tabular}{ll}
\hline $\begin{array}{l}\text { Cuál es la } \\
\text { evidencia }\end{array}$ & $\begin{array}{l}\text { Encontramos seis revisiones sistemáticas } \\
(2,3,4,5,6,7)\end{array}$ \\
$\begin{array}{l}\text { Véase matriz } \text { mincluyeron 17 estudios prima- } \\
\text { de evidencia }\end{array}$ & cuatro son ensayos aleatorizados $^{(13,14,21,23)}$. \\
$\begin{array}{l}\text { en Epistemo- } \\
\text { nikos más } \\
\text { abajo. }\end{array}$ & $\begin{array}{l}\text { Esta tabla y el resumen en general, se basan en es- } \\
\text { tos últimos, dado que los estudios observacionales }\end{array}$ \\
& no aumentaban la certeza de la evidencia existente \\
ni entregaban información adicional relevante.
\end{tabular}

Todos los ensayos incluyeron pacientes adultos con fracturas del reborde y/o piso orbitario sin restricción por edad ${ }^{(13,14,21,23)}$.

Tres ensayos reportaron que la distribución por sexo de los participantes presentó una mayor proporción de hombres $(91 \%)$ sobre mujeres $(9 \%)^{(13,14,23)}$. Un ensayo no reportó la distribución por sexo(21).

Qué tipo de pacientes incluyeron los estudios*
Todos los ensayos incluyeron pacientes con fracturas del complejo orbitocigomático ${ }^{(13,14,21,23)}$, mientras que un ensayo ${ }^{(13)}$ también incorporó pacientes con fractura de piso orbitario.

La mayoría de los ensayos excluyeron a los pacientes con fracturas conminutas del piso de órbita o reborde infraorbitario, así como pacientes con injuria extensa de tejidos blandos periorbitarios ${ }^{(13,21,23)}$. Además, un ensayo ${ }^{(21)}$ excluyó fracturas del complejo orbitocigomático con desplazamiento mayor a 5 $\mathrm{mm}$ y otro ${ }^{(14)}$ no reportó sus criterios de exclusión.

Todos los ensayos compararon el abordaje transconjuntival preseptal con el abordaje subciliar para el tratamiento de fracturas del piso de órbita y/o reborde infraorbitario ${ }^{(13,14,21,23)}$.

Qué tipo de intervenciones incluyeron los estudios*

Para lograr un mejor acceso y visualización del sitio de fractura, en todos los ensayos se realizaron cantotomías laterales como complemento al abordaje transconjuntival preseptal $\left.\right|^{(13,14,21,23)}$. Sin embargo, en un ensayo ${ }^{(13)}$ la cantotomía lateral fue realizada únicamente en aquellos participantes en que fue requerida según el criterio de los operadores.

Los ensayos reportaron múltiples desenlaces, los cuales fueron agrupados por las revisiones sistemáticas de la siguiente manera:

- Ectropión (eversión palpebral inferior)

- Entropión (inversión palpebral inferior)

- Complicaciones intra y postoperatorias (diplopía,

Qué tipo de desenlaces midieron parestesia transitoria, equimosis, exposición escleral, laceración del plato tarsal o laceración palpebral inferior)

- Resultado estético insatisfactorio

El seguimiento promedio de los ensayos fue de 13,5 semanas con un rango que fluctúa entre 6 y 24 se$\operatorname{manas}^{(13,14,21,23)}$

* La información sobre los estudios primarios es extraída desde las revisiones sistemáticas identificadas, no directamente desde los estudios, a menos que se especifique lo contrario.

\section{Resumen de los resultados}

La información sobre los efectos del abordaje transconjuntival preseptal comparado con el abordaje subciliar está basada en cuatro ensayos $^{(13,14,21,23)}$ que incluyeron 120 pacientes.

Todos los ensayos ${ }^{(13,14,21,23)}$ midieron el desenlace ectropión y complicaciones intra y postoperatorias (120 pacientes), tres ensayos $^{(13,14,23)}$ midieron el desenlace entropión (100 pacientes) y un ensayo(13) midió el desenlace resultado estético insatisfactorio (40 pacientes). Ninguna de las revisiones sistemáticas reportó el tiempo de realización del abordaje.

\section{El resumen de los resultados es el siguiente:}

- El abordaje transconjuntival preseptal en comparación con el abordaje subciliar podría disminuir el riesgo de ectropión (baja certeza de evidencia)

- El abordaje transconjuntival preseptal en comparación con el abordaje subciliar podría aumentar el riesgo de entropión (baja certeza de evidencia)

- El abordaje transconjuntival preseptal podría disminuir el riesgo de resultado estético insatisfactorio por parte del paciente (baja certeza de evidencia)

- No se encontraron estudios que evaluaran el desenlace tiempo de realización del abordaje.

- El abordaje transconjuntival preseptal comparado con el abordaje subciliar, probablemente disminuye el riesgo de complicaciones intra y postoperatorias, tales como diplopía, parestesia transitoria, equimosis, exposición escleral, laceración del plato tarsal y laceración del palpebral inferior. 


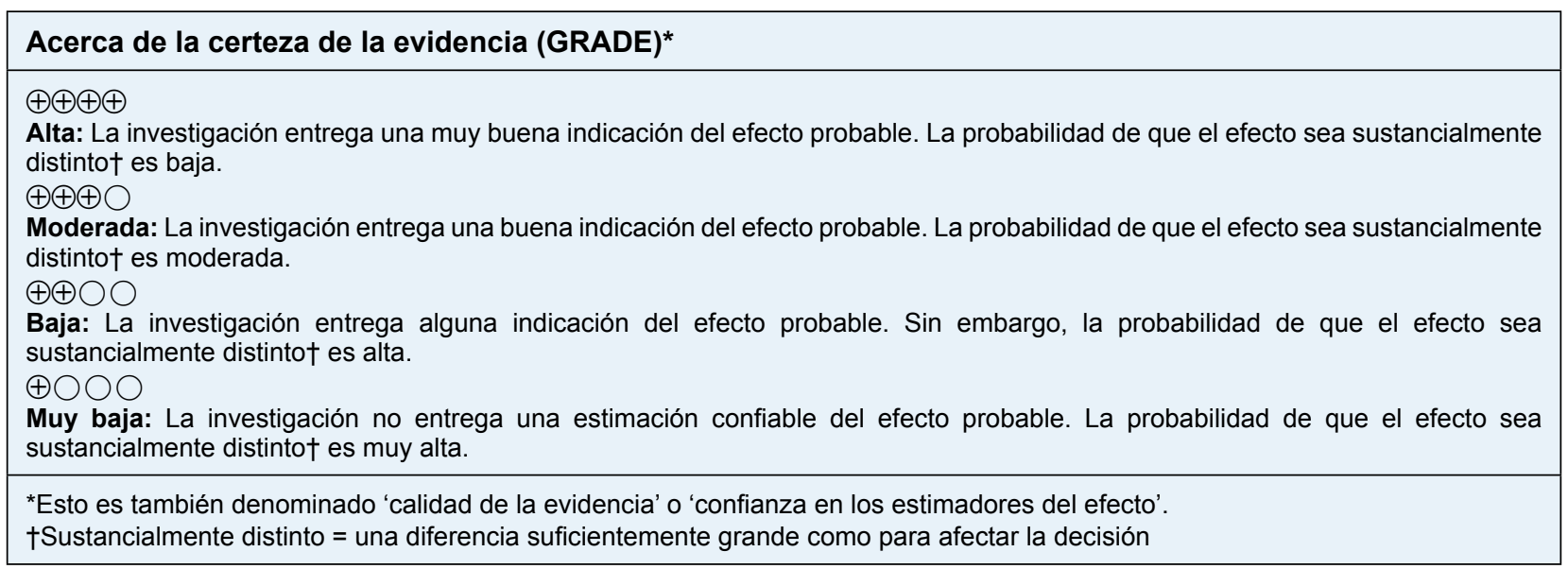

\section{Otras consideraciones para la toma de decisión}

\section{A quién se aplica y a quién no se aplica esta evidencia}

- Los resultados de este resumen son aplicables a pacientes adultos con fracturas de piso de órbita y/o reborde infraorbitario con un desplazamiento no mayor a $5 \mathrm{~mm}$

- Esta evidencia no se aplica a pacientes con fractura conminuta o daño extenso de los tejidos blandos periorbitarios.

\section{Sobre los desenlaces incluidos en este resumen}

- Los desenlaces incluidos en esta tabla son aquellos considerados críticos para la toma de decisiones de acuerdo a la opinión de los autores de este resumen y se encuentran en concordancia con las revisiones sistemáticas identificadas.

- Los autores consideran que un desenlace relevante para la toma de decisiones es el tiempo de realización del abordaje, el cual no fue medido por las revisiones sistemáticas.

\section{Balance daño/beneficio y certeza de la evidencia}

- El conjunto de evidencia podría mostrar un posible beneficio en la disminución del riesgo de ectropión y mejora en el resultado estético mediante el abordaje transconjuntival preseptal en comparación con el abordaje subciliar (certeza de evidencia baja). Además, el uso de abordaje transconjuntival preseptal probablemente disminuye el riesgo de desarrollar otras complicaciones.

- Por otro lado, el abordaje transconjuntival podría aumentar el riesgo de entropión (certeza de evidencia baja).

- Considerando lo anterior, el balance daño/beneficio podría estar a favor de la técnica de abordaje transconjuntival. Sin embargo, se considera que la toma de decisión debe tener en cuenta las características y preferencias de los pacientes, ya que factores como la edad podrían modificar el efecto de la intervención. Esto considerando los cambios fisiológicos propios de envejecimiento de los tejidos blandos periorbitarios, que podrían aumentar el riesgo de ectropión o entropión en pacientes de mayor edad ${ }^{(25)}$

\section{Consideraciones de recursos}

- Las revisiones sistemáticas no mencionan los costos asociados a cada intervención. Sin embargo, el abordaje transconjuntival preseptal podría presentar menores complicaciones postoperatorias que eventualmente se traduzcan en menores costos futuros.

- Sobre la base de la evidencia existente, faltan nuevos ensayos que midan el tiempo quirúrgico total, considerando desde la diéresis de los tejidos hasta la exposición de la fractura y desde finalizada la fijación hasta el cierre completo de los tejidos, si corresponde. Dado que el tiempo de utilización de pabellones quirúrgicos constituye un recurso limitado, se debe priorizar la utilización eficiente de éstos para resolver las fracturas adecuadamente en el menor tiempo posible.

- Debe considerarse que al llevar a cabo un abordaje transconjuntival se establece una relación directa entre el instrumental y la superficie del globo ocular. A diferencia del abordaje subciliar, el abordaje transconjuntival requiere para la disminución del riesgo de daño ocular la utilización de dispositivos como protectores corneales o esclerales que podrían aumentar los costos asociados a dicho abordaje.

\section{Qué piensan los pacientes y sus tratantes}

- Enfrentados a la evidencia presentada en este resumen, la mayoría de los pacientes y tratantes deberían inclinarse por el abordaje transconjuntival preseptal para el tratamiento quirúrgico de fracturas orbitarias. Esto considerando que de forma global, el abordaje transconjuntival presentó menores complicaciones que el abordaje subciliar. Sin embargo, debe considerarse en la toma de decisión la experiencia y comodidad del clínico con cada una de las técnicas ${ }^{(3)}$

- Podría existir una mayor preferencia por parte de los pacientes por el abordaje transconjuntival, ya que al contrario de los abordajes transcutáneos, no produce una cicatriz cutánea posterior a su ejecución, además de observarse resultados estéticos más favorables ${ }^{(13)}$.

- Por otro lado, algunos clínicos consideran que la utilización del abordaje subciliar para las fracturas del piso de la órbita otorgaría una mayor visibilidad sin la necesidad de una técnica quirúrgica complementaria como la cantotomía lateral en el caso de la utilización del abordaje transconjuntival preseptal $^{(1)}$. Por otro lado, según los estudios primarios revisados ${ }^{(13,14,21,23)}$, el tiempo de realización del abordaje subciliar en comparación con el abordaje transconjuntival preseptal con cantotomía lateral es menor.

\section{Diferencias entre este resumen y otras fuentes}

- Las conclusiones de este resumen coinciden en general con las de las revisiones identificadas respecto al riesgo de desarrollar ectropión, entropión y resultados estéticos insatisfactorios. Sin embargo, difieren al evaluar el desenlace otras complicaciones intra y post operatorias, dado que ellas concluyen que no existirían diferencias entre los abordajes ${ }^{(4,5,6)}$.

- Estas diferencias podrían explicarse debido a que las revisiones incorporaron en su análisis ensayos aleatorizados y estudios observacionales, lo que podría alterar los resultados obtenidos ${ }^{(26)}$. Además, ninguna de las revisiones evaluó la certeza de la evidencia de los desenlaces.

\section{¿Puede que cambie esta información en el futuro?}

- La probabilidad de que futura investigación cambie las conclusiones de este resumen es alta para algunos desenlaces (ectropión, entropión y resultado estético insatisfactorio) y moderada para otros (complicaciones intra y postoperatorias) debido a la incertidumbre de la evidencia existente.

- Luego de realizar una búsqueda en la International Clinical Trials Registry Platform de la Organización Mundial de la Salud y en la base de datos PROSPERO, no se identificaron registros de ensayos clínicos ni revisiones sistemáticas relacionados con la pregunta de investigación. 


\section{CÓMO REALIZAMOS ESTE RESUMEN}

Mediante métodos automatizados y colaborativos recopilamos toda la evidencia relevante para la pregunta de interés y la presentamos en una matriz de evidencia.
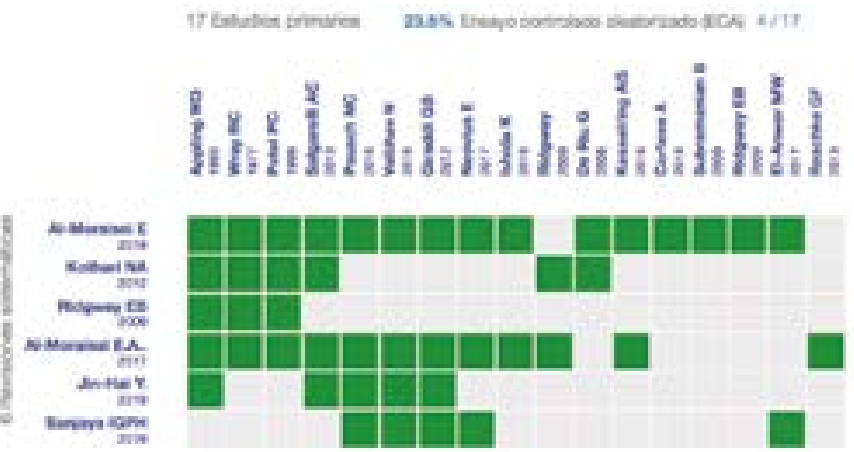

Figura 1. Matriz de evidencia "Abordaje transconjuntival comparado con abordaje subciliar para fracturas orbitarias".

Siga el enlace para acceder a la versión interactiva: Abordaje transconjuntival comparado con subciliar para fracturas orbitarias.

\section{NOTAS}

Si con posterioridad a la publicación de este resumen se publican nuevas revisiones sistemáticas sobre este tema, en la parte superior de la matriz se mostrará un aviso de "nueva evidencia". "Si bien el proyecto contempla la actualización periódica de estos resúmenes, los usuarios están invitados a comentar en la página web de Medwave o contactar a los autores mediante correo electrónico si creen que hay evidencia que motive una actualización más precoz." Por "Si bien el proyecto contempla la actualización periódica de estos resúmenes, los usuarios están invitados a comentar en la página web de International Journal of Interdisciplinary Dentistry o contactar a los autores mediante correo electrónico si creen que hay evidencia que motive una actualización más precoz".

Luego de crear una cuenta en Epistemonikos, al guardar las matrices recibirá notificaciones automáticas cada vez que exista nueva evidencia que potencialmente responda a esta pregunta.

Este artículo es parte del proyecto síntesis de evidencia de Epistemonikos. Se elabora con una metodología preestablecida, siguiendo rigurosos estándares metodológicos y proceso de revisión por pares interno. Cada uno de estos artículos corresponde a un resumen, denominado FRISBEE (Friendly Summary of Body of Evidence using Epistemonikos), cuyo principal objetivo es sintetizar el conjunto de evidencia de una pregunta específica, en un formato amigable a los profesionales clínicos. Sus principales recursos se basan en la matriz de evidencia de Epistemonikos y análisis de resultados usando metodología GRADE. Mayores detalles de los métodos para elaborar este FRISBEE están descritos aquí (http://dx.doi.org/10.5867/medwave.2014.06.5997)

La Fundación Epistemonikos es una organización que busca acercar la información a quienes toman decisiones en salud, mediante el uso de tecnologías. Su principal desarrollo es la base de datos Epistemonikos (www.epistemonikos.org).

\section{DECLARACIÓN DE CONFLICTOS DE INTERESES}

Los autores declaran no tener conflictos de intereses con la materia de este artículo.

\section{Bibliografía}

1. Kim HS, Jeong EC. Orbital Floor Fracture. Arch Craniofac Surg. 2016;17(3):111118.

2. Kothari NA, Avashia YJ, Lemelman BT, Mir HS, Thaller SR. Incisions for orbital floor exploration. J Craniofac Surg. 2012;23(Suppl 1):1985-1989.

3. Ridgway EB, Chen C, Colakoglu S, Gautam S, Lee BT. The incidence of lower eyelid malposition after facial fracture repair: a retrospective study and metaanalysis comparing subtarsal, subciliary, and transconjunctival incisions. Plastic and reconstructive surgery. 2009;124(5):1578-86.

4. Al-Moraissi E.A., Thaller S.R., Ellis E.. Subciliary vs. transconjunctival approach for the management of orbital floor and periorbital fractures: A systematic review and meta-analysis. Journal of Cranio-Maxillofacial Surgery. 2017;45(10):1647-1654.

5. Al-Moraissi E, Elsharkawy A, Al-Tairi N, Farhan A, Abotaleb B, Alsharaee Y, Oginni FO, Al-Zabidi A. What surgical approach has the lowest risk of the lower lid complications in the treatment of orbital floor and periorbital fractures? A frequentist network meta-analysis. Journal of cranio-maxillo-facial surgery : official publication of the European Association for Cranio-Maxillo-Facial Surgery. 2018;46(12):2164-2175. 6. Jin-Hai Y., Hong-Fei L., Hao L., Meng-Lin S., Qi-Hua X.. A Meta-analysis of complications of orbital blowout fracture by surgical approaches. International Eye Science. 2019;19(4):577-581.

7. Sanjaya IGPH, Hamid ARRH, Adnyana IMS, Purwanthi IGAP, Magdalena P, Mardhika PE. Risk of Lower Eyelids Malposition in Subciliary Compared to Transconjunctival Approach in Maxillofacial Fractures Management: A Systematic Review and Meta-Analysis. Open Access Maced J Med Sci. 2019;7(17):2953-2957.

8. Appling WD, Patrinely JR, Salzer TA. Transconjunctival approach vs subciliary skinmuscle flap approach for orbital fracture repair. Archives of otolaryngology--head \& neck surgery. 1993;119(9):1000-7.

9. Wray RC, Holtmann B, Ribaudo JM, Keiter J, Weeks PM. A comparison of conjunctival and subciliary incisions for orbital fractures. British journal of plastic surgery. 1977;30(2):142-5

10. Patel PC, Sobota BT, Patel NM, Greene JS, Millman B. Comparison of transconjunctival versus subciliary approaches for orbital fractures: a review of 60 cases. The Journal of cranio-maxillofacial trauma. 1998;4(1):17-21.

11. Salgarelli AC, Bellini P, Landini B, Multinu A, Consolo U. A comparative study of different approaches in the treatment of orbital trauma: an experience based on 274 cases. Oral and maxillofacial surgery. 2010;14(1):23-7.

12. Pausch NC, Sirintawat N, Wagner R, Halama D, Dhanuthai K. Lower eyelid complications associated with transconjunctival versus subciliary approaches to orbital floor fractures. Oral and maxillofacial surgery. 2016;20(1):51-5.

13. Vaibhav N, Keerthi R, Nanjappa M, Ashwin DP, Reyazulla MA, Gopinath AL, Ghosh A. Comparison of 'sutureless' Transconjunctival and Subciliary Approach for Treatment of Infraorbital Rim Fractures: a Clinical Study. Journal of maxillofacial and oral surgery. 2016;15(3):355-362.
14. Giraddi GB, Syed MK. Preseptal transconjunctival vs. subciliary approach in treatment of infraorbital rim and floor fractures. Annals of maxillofacial surgery. 2012;2(2):136-40.

15. Neovius E, Clarliden S, Farnebo F, Lundgren TK. Lower Eyelid Complications in Facial Fracture Surgery. The Journal of craniofacial surgery. 2017;28(2):391-393.

16. Ishida K. Evolution of the surgical approach to the orbitozygomatic fracture: From a subciliary to a transconjunctival and to a novel extended transconjunctival approach without skin incisions. Journal of plastic, reconstructive \& aesthetic surgery : JPRAS. 2016;69(4):497-505.

17. Ridgway, E. B., Chen, C., Colakoglu, S., Gautam, S., \& Lee, B. T. The incidence of lower eyelid malposition after facial fracture repair: a retrospective study and metaanalysis comparing subtarsal, subciliary, and transconjunctival incisions. Plastic and Reconstructive Surgery. 2009;124(5):1578-1586.

18. De Riu G, Meloni SM, Gobbi R, Soma D, Baj A, Tullio A. Subciliary versus swinging eyelid approach to the orbital floor. Journal of cranio-maxillo-facial surgery : official publication of the European Association for Cranio-Maxillo-Facial Surgery 2008:36(8):439-42.

19. Kesselring AG, Promes P, Strabbing EM, van der Wal KG, Koudstaal MJ. Lower Eyelid Malposition Following Orbital Fracture Surgery: A Retrospective Analysis Based on 198 Surgeries. Craniomaxillofacial trauma \& reconstruction. 2016;9(2):109-12.

20. Cortese A, Caggiano M, Carlino F, Pantaleo G. Zygomatic fractures: Technical modifications for better aesthetic and functional results in older patients. International journal of surgery (London, England). 2016;33 Suppl 1:S9-S15.

21. Subramanian B, Krishnamurthy S, Suresh Kumar P, Saravanan B, Padhmanabhan M. Comparison of various approaches for exposure of infraorbital rim fractures of zygoma. Journal of maxillofacial and oral surgery. 2009;8(2):99-102.

22. Ridgway EB, Chen C, Lee BT. Acquired entropion associated with the transconjunctival incision for facial fracture management. The Journal of craniofacial surgery. 2009;20(5):1412-5.

23. El-Anwar MW, Elsheikh E, Hussein AM, Tantawy AA, Abdelbaki YM. Transconjunctival versus subciliary approach to the infraorbital margin for open reduction of zygomaticomaxillary complex fractures: a randomized feasibility study. Oral and maxillofacial surgery. 2017;21(2):187-192.

24. Raschke GF, Rieger UM, Bader RD, Schaefer O, Guentsch A, Schultze-Mosgau S. Transconjunctival versus subciliary approach for orbital fracture repair--an anthropometric evaluation of 221 cases. Clinical oral investigations. 2013;17(3):93342.

25. Salvi SM, Akhtar S, Currie Z. Ageing changes in the eye. Postgrad Med J. 2006;82(971):581-587.

26. Bun RS, Scheer J, Guillo S, Tubach F, Dechartres A. Meta-analyses frequently pooled different study types together: a meta-epidemiological study. J Clin Epidemiol 2020;118:18-28. 